\title{
ROLE OF SERUM D DIMER LEVELS IN ACUTE ISCHEMIC STROKE
}

\author{
Dr Puneet Patil ${ }^{1}$, Dr Manoj Patel ${ }^{2}$, Dr Aamera Sait ${ }^{3}$ \\ ${ }^{1}$ Associate Professor, Dept. of General Medicine, ACPM Medical College, Dhule \\ ${ }^{2}$ Assistant Professor, Dept. of General Medicine, ACPM Medical College, Dhule \\ ${ }^{3}$ Registrar, Critical Care Medicine, Manipal Hospital, Banglore
}

Article Info: Received 27 October 2021; Accepted 10 December 2021

DOI: https://doi.org/10.32553/ijmbs.v5i12.2365

Corresponding author: Dr Manoj Patel

Conflict of interest: No conflict of interest.

\begin{abstract}
Background: Acute ischemic stroke has been known to be a severely debilitating affliction with a steep disability and co morbidity curve. Various tools and methods have been attempted to ascertain if the diagnosis and prognosis of stroke can be achieved faster as it aids in effective management as well as reduces the resultant damage to the brain. Aim: The present study was designed to assess if serum D Dimer levels can be used as a marker as well as a volumetric determinant of stroke. Methods: The study involved a patient pool of 50 patients and corresponding controls who were matched for age and gender. The data collected included demographic data, baseline clinical parameters, serum D Dimer levels and a radiological assessment of the volume of infract. Findings: The study revealed that a relationship exists between the levels of circulating D Dimer and DW MRI weighted images showing infarcts. The relationship was direct in nature. Conclusion: D Dimer levels can provide a estimate of the area under infarction and thus prove to be a prognostic as well as diagnostic marker.
\end{abstract}

Keywords: Acute ischaemic Stroke, D Dimer, Volumetric Association

\section{Introduction}

A by-product of fibrin degradation in thrombi is known as $D$-Dimer. It is in use as a current marker for venous thromboembolism in suspected cases and has been clinically utilized as a marker in such scenarios due to its relative resistance to false positivity, a stable and detectable half-life and its persistence in clinically relevant conditions. (1) The values of circulating $D$ Dimer is an indicator of the amount of fibrin degradation. In plasmin mediated degradation of several fibrin based products, the values of D Dimer are elevated and thus a intrinsic value denotes actual degradation status. (2) Studies have hypothesized that an elevated D Dimer level in systemic circulation can be a potential indication of increased coagulability of blood, clot formation or fibrin formations which can be further seen in symptoms/ conditions leading up to coronary heart disease. (3) The direct association between circulating D Dimer levels and heart disease in noted in many studies and has now been integrated in clinical practice when possible. $(3,4)$. D Dimer elevations have also been seen in multitude of similarly occlusive states such as venous sinus thrombosis, acute pulmonary emboli, hemorrhagic stroke, ischemic stroke etc. The use of D Dimer in neurological cases has been gaining repute and evidence based research has postulated the efficacy of D Dimer as a prognostic marker as well as an outcome predictor in case of acute ischemic stroke and traumatic brain injury. (5-10) Unfortunately, little or no research has been conducted in the sub population in Rural Maharashtra on efficacy of D Dimer and its resultant value in cases of Acute Ischemic Stroke. With this aim, a study was formed to find an association between levels of D Dimer and stroke volume and severity among patients of acute ischaemic stroke in Rural Maharashtra.

\section{Materials and Methods:}

The present study was a cross-sectional prospective case control study which was conducted over a period of 9 months (March to November 2020) at ACPM Medical College, Dhule, Maharashtra. Institutional ethical clearance was obtained prior to starting of the study. The study employed a study pool of 50 adult subjects who had experienced acute ischemic stroke for the first time. A age and gender appropriate 
control group without the diagnosis of stroke was also used for comparative evaluation. The study entailed obtaining a written informed consent from the patient/ guardian/relative for inclusion in the study. The data collected included demographic data (age and sex) and history of conventional vascular risk factors were obtained. Routine blood and biochemical tests, ECG, and a baseline brain CT/MRI scan were performed in all patients at admission. Stroke severity was assessed on admission using the National Institutes of Health Stroke Scale (NIHSS, the NIHSS score ranges from 0 to 34 and higher values reflect more severe neurological damage) (11) by a neurologist. Patients' blood samples were collected in the ward bedside in 5-ml vacuum tubes containing 0.5 $\mathrm{ml}$ buffered sodium citrate. D-dimer concentration was measured with a particle-enhanced, immunoturbidimetric assay. The resultant data was noted in an MS Excel Data sheet and subjected to statistical analysis in co-ordination with institutional statistician.

\section{Observations:}

The study included a total of 50 subjects and 50 controls. The demographic parameters and risk factors were as depicted in table 01.

The values of systolic blood pressure and Glucose showed a statistically significant difference in the test and control group. In terms of correlation of D Dimer values and NIHSS grading. It was observed that the severity of stroke had a correlation with D Dimer elevations (Fig 01)

In terms of stroke volume, patients were found to have a positive correlation between levels of D Dimer and infarct volume as determined by DWI-MRI analysis. This led to the inference that there exists a statistically significant correlation between the values of D Dimer in circulation and extent of damage due to stroke.

\section{Discussion:}

The present study showed that circulating D Dimer values are significantly elevated in case of acute ischemic stroke when compared with age and gender matched controls. Additionally, it was seen that $D$ Dimer levels were increased with an increase in severity of stroke as determined by NIHSS scoring system. The scoring severity was corelating with $D$ Dimer values even after management protocols were implemented. A similarly significant correlation was seen in stroke volume and D Dimer levels depicting that levels of D Dimers may elevate in corresponding with the volume of brain undergoing infarction. These findings are in concurrence with similar studies wherein the authors attempted to understand a relationship between D Dimer and acute ischemic stroke in case control format. $(14,15)$ Some studies reported that variations in D Dimer levels occur when age and gender comes into play. This is also in concurrence with our study, wherein such features were noted. (16) Elevated D Dimer values were positively linked with stroke severity in our study and this is in concurrence with study by Berge F et al. (17). However we are limited by the fact that outcome was not a measured variable in the study. We found that $D$ Dimer was elevated proportionally to the area of the infarct. This is in similarity to similar studies by various authors who reported that not only is D Dimer values associated with a brain infarct, but it also has a statistical correlation with the volume of infarct. $(18,19)$

\section{Conclusion:}

It was determined from the present study that an elevated D Dimer value is indicative of a similarly elevated stroke severity. There exists a positive correlation between stroke severity and circulating $D$ Dimer values which can be utilized as a marker for prognosis. Additionally, the area of infarction can also be assessed using $D$ Dimer thus can be an acute stage marker for outcome. The present study is limited by the small sample size. A larger long term study with a wider distribution of patient demographics can aid in determining the efficacy of this marker.

\section{References:}

1. Lowe GD. Fibrin D-dimer and cardiovascular risk. In Seminars in vascular medicine 2005 Dec (Vol. 5, No. 04, pp. 387-398). Copyright @ 2005 by Thieme Medical Publishers, Inc., 333 Seventh Avenue, New York, NY 10001, USA..

2. Lowe GD, Rumley A. Use of fibrinogen and fibrin D-dimer in prediction of arterial thrombotic events. Thrombosis and haemostasis. 1999;82(08):667-72.

3. Lippi G, Filippozzi L, Montagnana M, Salvagno GL, Guidi GC. Diagnostic value of D-dimer measurement in patients referred to the emergency department with suspected 
myocardial ischemia. Journal of thrombosis and thrombolysis. 2008 Jun;25(3):247-50.

4. Barber M, Langhorne P, Rumley A, Lowe GD, Stott DJ. D-dimer predicts early clinical progression in ischemic stroke: confirmation using routine clinical assays. Stroke. 2006 Apr 1;37(4):1113-5.

5. Meng $R$, Wang $X$, Hussain $M$, Dornbos III $D$, Meng L, Liu Y, Wu Y, Ning M, Ferdinando S B, Lo EH, Ding $Y$. Evaluation of plasma D-dimer plus fibrinogen in predicting acute CVST. International Journal of Stroke. 2014 Feb;9(2):166-73.

6. Chiu CC, Li YN, Lin L, Hsiao CT, Hsiao KY, Chen IC. Serum D-dimer as a predictor of mortality in patients with acute spontaneous intracerebral hemorrhage. Journal of Clinical Neuroscience. 2012 Jun 1;19(6):810-3.

7. Montaner J, Perea-Gainza el ado $P$ ib hac $n$ P osell intana Palacios olina I are - ab n. Etiologic diagnosis of ischemic stroke subtypes with plasma biomarkers. Stroke. 2008 Aug 1;39(8):2280-7.

8. Hiltunen S, Putaala J, Haapaniemi E, Salonen O, Tatlisumak T. D-dimer and clinicoradiologic features in cerebral venous thrombosis. Journal of the neurological sciences. 2013 Apr 15;327(12):12- 4.

9. Hudak $A M$, Caesar RR, Frol AB, Krueger K, Harper CR, Temkin NR, Dikmen SS, Carlile M, Madden C, Diaz-Arrastia R. Functional outcome scales in traumatic brain injury: a comparison of the Glasgow Outcome Scale (Extended) and the Functional Status Examination. Journal of neurotrauma. 2005 Nov 1;22(11):1319-26.

10. Ungerstedt JS, Grenander $\AA$, Bredbacka $S$, Blombäck $M$. Clotting onset time may be a predictor of outcome in human brain injury: a pilot study. Journal of neurosurgical anesthesiology. 2003 Jan 1;15(1):13-8.
11. Brott T, Adams HP Jr, Olinger CP, Marler JR, Barsanl WG, et al (1989) Measurements of acute cerebral infarction: A clinical examination scale. Stroke 20: 864-870.

12. Adams HP, Bendixen BH, Kappelle L, Biller J, Love BB (1993) Classification of subtype of acute ischemic stroke. Definitions for use in a multicenter clinical trial. TOAST. Trial of Org 10172 in Acute Stroke Treatment. Stroke 24: 35-41.

13. Bamford J, Sandercock P, Dennis M, Burn J, Warlow C (1991) Classification and natural history of clinically identifiable subtypes of cerebral infarction. Lancet 337: 1521-1526.

14. Kataoka S, Hirose G, Hori A, Shirakawa T, Saigan T (2000) Activation of thrombosis and fibrinolysis following brain infarction. J Neurol Sci 181: 82-88.

15. Tombul T, Atbas C, Anlar O (2005) Hemostatic markers and platelet aggregation factors as predictive markers for type of stroke and neurological disability following cerebral infarction. J Clin Neurosci 12: 429-434.

16. Carcaillon L, Gaussem P, Ducimetiere P, Giroud M, Ritchie K, et al (2009) Elevated plasma fibrin Ddimer as a risk factor for vascular dementia: the Three- City cohort Study. J Thromb Haemost 7: 1972-1978.

17. Berge E, Friis $P$, Sandset $P$ (2001) Hemostatic activation in acute ischemic stroke. Thromb Res 101: 13-21.

18. Matsumoto M, Sakaguchi M, Okazaki S, Furukado S, Tagaya M, et al (2013) Relationship between plasma (D)-dimer level and cerebral infarction volume in patients with nonvalvular atrial fibrillation. Cerebrovasc Dis 35: 64-72.

19. Park YW, Koh EJ, Choi HY (2011) Correlation between serum $\mathrm{D}$-dimer level and volume in acute ischemic stroke. J Korean Neurosurg Soc 50: 8994. 